\title{
Lockdown Diet: A Recipe for Impending Surge of Dental Caries Cases in Children?
}

Aditi Kapur ${ }^{1}$, Richa Mishra ${ }^{2}$

\begin{abstract}
The ongoing lockdown situation due to coronavirus disease-2019 (COVID-19) pandemic is having its influence on the physical and mental health of individuals in varied ways, with a majority of the population struggling with a change in patterns of day-to-day chores, routine, and behavior. This change of pattern is also observed in diet and nutrition of people, particularly children, who are facing this unique challenge. The influence of dietary habits on overall health in general and oral health in particular can never be overemphasized. The changes in dietary patterns among children during these times may, therefore, have an impact on dental caries cases in the near future.

Keywords: Children, COVID-19, Dental caries, Diet, Lockdown.

Journal of Postgraduate Medicine, Education and Research (2020): 10.5005/jp-journals-10028-1377
\end{abstract}

\section{INTRODUCTION}

Lockdown life has become the "new normal" as every individual has rewoven the fabric of his or her lifestyle around the recent coronavirus disease-2019 (COVID-19) pandemic. This stems from awareness about the importance of confinement and physical distancing among a great number of people, and government regulations for the others. Resilience at both individual and community level has been suggested to be the greatest weapon in fighting the present pandemic situation, requiring psychological as well as behavioral adaptations. ${ }^{1}$

The adjustments during the present times are mostly developing some adaptive habits to handle ongoing changes and some maladaptive habits to cope with them. The delicate balance between these habits, accumulating over time is likely to shape the individual's physical, mental, and social health in the near future. These adaptive changes are also visible in habits involving dietary and physical activity patterns. Lockdown and confinement renders an individual susceptible to a sedentary lifestyle with very low energy expenditure, as most of the activities are performed in a sitting or supine position and could also lead to irregular eating patterns and frequent snacking.

A recent Italian online survey conducted on 3,533 individuals, aged 12-87 years confirmed such a change in dietary patterns during the coronavirus pandemic, bringing to notice an increase in snacking frequency in-between meals and during late hours of night, before sleeping. It was emphasized that this change in habits was greater among the younger age groups. Though the consumption of processed food and carbonated drinks had decreased owing to less availability, there was a notable surge in the consumption of homemade sweets. ${ }^{2}$ Similarly, a study from Poland also reported an increase in snacking frequency in $52 \%$ of 1,097 adults enrolled for the study. ${ }^{3}$ However, an increase in intake of dairy, meat, and processed food was noted in this study in contrast to the aforementioned Italian data, where homemade sweets were consumed the most.

The causes of changes in dietary patterns are decided in general by the degree of lockdown imposed by individual governments across the globe and hence, the availability or unavailability of
1,2Paediatric Dentistry Unit, Oral Health Sciences Centre, Postgraduate Institute of Medical Education and Research, Chandigarh, India

Corresponding Author: Aditi Kapur, Paediatric Dentistry Unit, Oral Health Sciences Centre, Postgraduate Institute of Medical Education and Research, Chandigarh, India, Phone: +91 9815966348, e-mail: draditikmalhotra@gmail.com

How to cite this article: Kapur A, Mishra R. Lockdown Diet: A Recipe for Impending Surge of Dental Caries Cases in Children? J Postgrad Med Edu Res 2020;54(3):100-102.

Source of support: Nil

Conflict of interest: None

certain food items. However, there are other determining factors which may lead to maladaptive eating habits among individuals. Evidence has shown dietary habits to be affected by stress, distress, and emotional status of an individual and elevated levels of distress are associated with unhealthy patterns of eating and poor quality of the diet. ${ }^{4}$ The recent pandemic has resulted in an atmosphere of anxiety and fear, affecting every individual across the globe, by varying degrees. In addition, there is another set of population which considers itself a victim of boredom during the lockdown. A series of experimental studies have proved the changes in dietary patterns in subjects as a means of distancing themselves away from the awareness of boredom. ${ }^{5}$ Dietary maladaptation due to any reason is bound to have short-term and lasting influence upon the overall health of individuals and oral health is no exclusion in this scenario.

\section{The Challenges}

The anticipations regarding an impending surge of caries is not only an obvious hypothesis, but is backed by decades of studies in which role of diet in carious process have been studied in various settings.

\section{Facts Backing this Anticipation}

An increased frequency of food consumption, frequent snacking, and inadvertent intake of sugary food are known risk factors for dental caries. Literature reveals an increment of one carious 
lesion for every $25 \mathrm{~g}$ sugar availability a day among 12-year-olds and $50 \mathrm{~g}$ a day for 6-year-olds. ${ }^{6}$ However, it is the frequency of consumption of sugary items that has a more significant relationship with the caries experience rather than the amount, as shown by certain classical studies in the past, thereby supporting the fact that in-between snacking needs to be reduced. ${ }^{7}$ The logistic regression coefficients for both these parameters, however, vary in a limited range. ${ }^{7}$ Dental caries is a dynamic process wherein lesion formation occurs resulting from repetitive cycles of demineralization and remineralization. Newer carious lesions would initially present only as demineralization of enamel, thereby making their identification difficult without an expertise in the field. This is followed by cavitation in due course of time depending upon the balance of risk factors and preventive factors which are involved in the process. There exists a delicate balance in the $\mathrm{pH}$ shifts of saliva, which when persisting over a period of time, favors demineralization of hard tissue. ${ }^{8}$ The time is generally accepted to be around 45 minutes and the critical salivary pH as 5-5.5. ${ }^{7}$ Hence, subsequent sugar exposures at a short intervals would not allow enough time for the natural defenses in saliva to tip mineral balance to aid in remineralization. This theoretical concept is clearly evident in children who have a habit of eating sweets in pieces over prolonged hours or have a habit of pouching food. The situation worsens with the sugary nature and stickiness of food items. It has become rather difficult to obtain a linear or log-linear relationship between sugar intake and caries in the past few decades, owing to the modification of the same by the usage of fluoride, especially through fluoridated toothpastes available in different concentrations of fluoride. However, studies still show sugar as a causative agent in caries experience through the postfluoridation era till date. ${ }^{7}$

The present situation is being compared to others epidemics and World Wars. The present coronavirus pandemic has a greater influence in terms of the number of lives affected and duration for which it has been prevailing now, unlike the other epidemic in recent times. There was a significant decrease in caries incidence in the post-World War II era owing to reduced availability of sugar. ${ }^{6}$ However, the situation in the current pandemic is different. There is availability of not only ration but also processed food depending upon the degree of lockdown in individual countries. Therefore, the imaginary situation in which a child at home, with schools shut down and restrictions on playing outside, has high chances to turn to favorite food as a source of comfort. Even if there is a dearth of literature on this regard, this hypothetical scenario is very feasible in any household setting.

The situation becomes even worse when the child is already in a high-risk category for development of caries. These also include children with other systemic disorders, such as the ones with long-term hospitalization or medication, the ones undergoing chemo- and/or radiotherapy, and the ones with chronic illnesses that either influence salivary parameters of individuals or impair the maintenance of oral hygiene. Children undergoing orthodontic therapy or children with syndromes affecting the maxillofacial region and children with cleft lip and palate are also in high-risk categories. In addition, a vast majority of children with untreated open carious lesions are also included in this category.

\section{Where are We Lacking?}

Fluoridated toothpastes have played the most important role in caries prevention over decades, due to their cost-effectiveness and widespread availability. But, even brushing twice daily with fluoridated toothpastes and regular flossing should not be counted as an excuse for carrying out maladaptive dietary practices. Moreover, dental awareness in a developing country like ours has not reached the level at which every child and parent knows the importance of nighttime brushing. Water fluoridation in an equitable manner is lacking and so are the other sources of fluoride, such as fluoridated milk or salt. Newer remineralizing agents have been shown to have additive benefits in caries protection, but lack a widespread use owing to awareness and cost factors. Ecological approaches of caries prevention, such as the use of probiotics or xylitol chewing gums, also do not have a diverse usage. Also, the knowledge of other herbal methods of caries prevention either still lack evidence for usage or are native to other countries, where research is being carried out on them. Also, there does not exist any uniform preventive program throughout our country to educate and treat children and their parents about oral hygiene measures. Considering all these factors, dietary modifications can prove to be a beneficial protective modality for caries prevention in a country like ours. This is not only of importance considering the lockdown, but the fact that countries with recent urbanization and mass migration of people to cities are more likely to have shifts of dietary patterns to a refined packaged diet.

\section{Add-on Challenges Due to COVID-19}

It has already been months through the lockdown when dental units had to be shut to avoid aerosol generation, thereby leading to an accumulation of surplus cases of dental caries. The symptomatic ones are being managed with either with analgesics or interim procedures. ${ }^{9}$ But, there is a vast majority of population that does not have easy access to dental facilities during lockdown. All of these factors already pose a great number of impending cases, which would be further taken care of according to their severity.

\section{Recommendations}

Considering all these factors, it is most important to rely upon preventive measures at a very domestic level. The involvement of parents with their children in tough times like these is of great importance. It is important to understand that the lockdown is having its influence upon the mental health of children as well. ${ }^{10} \mathrm{~A}$ child who feels neglected is more likely to indulge in other habits for comfort, e.g., comfort food. At the same time, it is also not advisable to over indulge with children and pamper them with savories. Hence, the understanding of proper meals and healthy snacking is important.

Foremost, it is important to start the day with a healthy breakfast. Studies have shown the effect of skipping breakfast on the release of hormones, such as ghrelin and leptin, and the urge to keep snacking until it is time for the next meal. A recent study has shown that a low breakfast frequency per week was related to caries experience in 9- and 15-year-olds. ${ }^{11}$ Avoidance of in-betweenmeals snacking or replacing sugary snacks with healthier ones, such as nuts, fruits, homemade yogurt, and wholegrain cereals, is advisable. Although it is not possible to consume fruits and vegetables without releasing the intrinsic sugars, it is always advisable to replace other foods high in free sugars with fresh fruits. An important component of diet for children in our country is milk which has protective effects by the virtue of certain proteins like casein. However, milk with added sugars when retained in the mouth for longer durations, such as prolonged use of baby bottle, 
has severe detrimental effects on dentition of young children. It seems appropriate to acknowledge the fact that it would not be possible to check $\mathrm{pH}$ changes after consumption of any food item in a household scenario even with the use of $\mathrm{pH}$ strips. Therefore, it is wise to weigh the intrinsic benefit and harm and food item might cause to the dentition by the virtue of its stickiness, type of sweetness, amount and frequency of consumption, thus making smart decisions for the health of the child.

In an ideal situation, every child should have a dietary analysis performed by a pediatric dentist and receive customized advice based on their general health, geographic, and socioeconomic conditions. But that seems to be a distant goal. Hence, a diet beneficial for both general and dental health is one that has less amounts of free sugars, saturated fat and salts, good amounts of vegetables, fresh fruits, nuts and seeds, wholegrain carbohydrates with modest amounts of legumes, fish, poultry and plenty of fluids preferably water and milk, while limiting the consumption of sugar-sweetened beverages. ${ }^{5}$ As for sugar consumption, the WHO strongly recommends limiting added sugar intake to $<10 \%$ of total energy, with an added conditional recommendation of further reduction of the same to $5 \% .{ }^{12}$ For caries prevention, it is generally advised to limit sugar intake to twice with meals and once in-between.

At this point, it needs to be noted that there are no studies regarding the dental and oral effects of lifestyle changes because such a degree of lockdown could not be traced in literature. Moreover, very few studies on changes in lifestyle and nutrition exist. Since the present situation is new to the present generation of healthcare professionals, such studies are the need of the hour to brace ourselves for the "after-effects" of what the pandemic might bring.

\section{Conclusion}

It is always disheartening for a pediatric dentist to find themselves surrounded by children having such carious lesions which would have been evaded only if these children and their parents received proper oral care advice at the right time. The present lockdown situation has further increased the distance between healthcare facilities and patients, with services being limited to teleconsultation and emergency care. Since to be able to provide preventive advice on a one-to-one basis is difficult currently, regulatory advices being circulated need to be reformulated to address issues related to optimum diet and nutrition as well. The present race of humans would be considered truly successful in surviving this pandemic, when we survive with minimum adverse effects.

\section{References}

1. Naja F, Hamadeh R. Nutrition amid the COVID-19 pandemic: a multilevel framework for action. Eur J Clin Nutr 2020;74:1117-1121. DOI: 10.1038/s41430-020-0634-3.

2. Di Renzo L, Gualtieri P, Pivari F, et al. Eating habits and lifestyle changes during COVID-19 lockdown: an italian survey. J Transl Med 2020;18(1):229. DOI: 10.1186/s12967-020-02399-5.

3. Sidor A, Rzymski P. Dietary choices and habits during COVID-19 lockdown: experience from Poland. Nutrients 2020;12(6):1657. DOI: 10.3390/nu12061657.

4. Anton SD, Miller PM. Do negative emotions predict alcohol consumption, saturated fat intake, and physical activity in older adults? Behav Modif 2005;29(4):677-688. DOI: 10.1177/0145445503261164.

5. Moynihan AB, van Tilburg WAP, Igou ER, et al. Eaten up by boredom: Consuming food to escape awareness of the bored self. Front Psychol 2015;6:369. DOI: 10.3389/fpsyg.2015.00369.

6. Sheiham A, James WP. A reappraisal of the quantitative relationship between sugar intake and dental caries: the need for new criteria for developing goals for sugar intake. BMC Public Health 2014;14:863. DOI: 10.1186/1471-2458-14-863.

7. van Loveren C. Sugar restriction for caries prevention: amount and frequency. Which is more important? Caries Res 2019;53(2):168-175. DOI: $10.1159 / 000489571$.

8. Featherstone JDB. The caries balance: the basis for caries management by risk assessment. Oral Health Prev Dent 2004;2(Suppl 1): 259-264.

9. Ather A, Patel B, Ruparel NB, et al. Coronavirus disease 19 (COVID-19): implications for linical dental care. J Endod 2020;4(5):584-595.

10. Mental health and wellbeing of children and families impacted by COVID 19 [Internet]. [cited 2020 Jun 21]. Available from: https://www. unicef.org/northmacedonia/mental-health-and-wellbeing-childrenand-families-impacted-covid-19.

11. Dusseldorp E, Kamphuis M, Schuller A. Impact of lifestyle factors on caries experience in three different age groups: 9, 15, and 21-yearolds. Community Dent Oral Epidemiol 2015;43(1):9-16. DOI: 10.1111/ cdoe.12123.

12. Moynihan P, Makino $Y$, Petersen PE, et al. Implications of WHO guideline on sugars for dental health professionals. Community Dent Oral Epidemiol 2018;46(1):1-7. DOI: 10.1111/cdoe.12353. 